it can be screwed to a sampling pipe. Its inner surface must be perfectly smooth so that there is no chance for accumulations of dust before the filter is reached. Part 4 is a loose nut which holds Parts $\mathbf{I}$ and 3 together while in use.

The apparatus may be used in any position but the preferred arrangement is horizontal. When the gas to be filtered contains moisture the filtering device must be heated to about $100^{\circ} \mathrm{C}$. by enclosing it in an asbestos-lined box, termining the amount of precipitate as copper, cuprous and cupric oxides, the following method was adopted since it proved the most satisfactory and yielded the best of results if reasonable attention was paid to details. It might be said, further, that as such work of ten has to be done at odd times it is desirable to maintain a supply of sugar tubes' and only to titrate when there are a number of tests on hand. By exercising a little care the same tubes can be used repeatedly without change of felt.
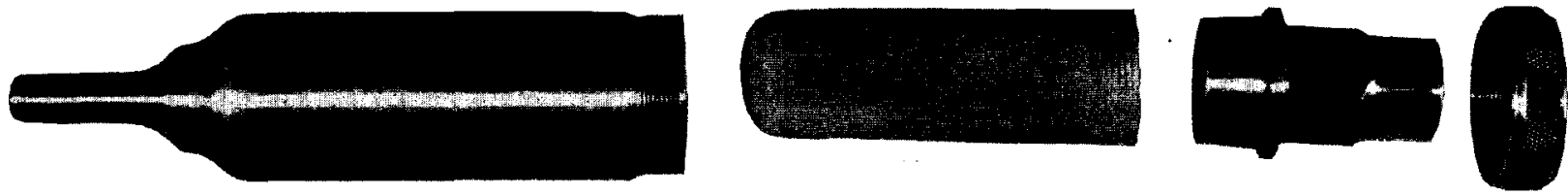

Fig. 1.

heated with an electric light, or by any other available means. The nipple on the exit end of Part I is provided with threads so that it can be removed and replaced with aluminium tubes, containing a dehydrating agent, in case it is desired to determine moisture in the gas (see Fig. 2).

The advantages of this apparatus as to simplicity are easily seen. Gravity seems to cause the heavy particles of dust to settle on the bottom of the shell, while the lighter particles form a porous coating on the upper surface. This action prevents the filter paper from becoming clogged as
The process consists of heating an aliquot of the "sugar solution with the mixed Allihn's solution (30 cc. of "white," $30 \mathrm{cc}$. of "blue" and $60 \mathrm{cc}$. of water) and filtering by aid of suction through a sugar tube with an asbestos felt supported by glass wool. The cuprous precipitate is transferred to the tube, washed with hot water until free from alkali and then with alcohol. The copper is dissolved in $5 \mathrm{cc}$, of concentrated nitric acid, thoroughly washediwith hot water, and the filtrate run into an Erlenmeyer flask by means of suction. The solution is evaporated to small

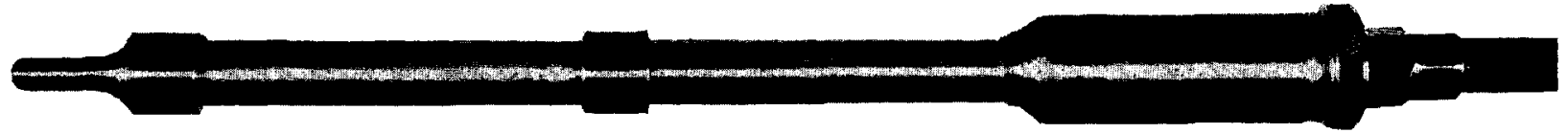

Fig. 2.

long astheat enough is applied to keep the paper dry. About twice as large samples of gas can be passed through this apparatus as through any other we have ever tried. We have also found the number of grains of dust per cu. ft. to be higher when determined by this apparatus than with the others.

Thus far the device has been used for filtering blast furnace gas only, but there is no reason why it cannot be used to filter air or any other gas. The apparatus described is now being made and sold by $E$. $H$. Sargent \& Co., whom I desire to thank for the loan of that shown in the illustrations.

I. A. Touzalin.

LABORATORY OF ILLINOIS STEEL CO., So. ChICAGo, ILL.

[From the Department of Plant and Animal Chemistry, MassaCHUSETTS AGRICULTURAI EXPERIMENT STATION.]

\section{VOLUMETRIC DETERMINATION OF COPPER IN SUGAR ANALYSIS. ?}

The co-operation of the laboratory in the experiments conducted by other departments of the Station has often rendered necessary quantitative determinations of reducing sugar, sucrose, lactose and starch in a variety of products. The final step in every case is the determination of the cuprous oxide precipitated from Allihn's solution by the reducing action of the sugar. After a rather long study' of different methods of filtration and the various ways of de-

${ }^{1}$ An adaptation of the Low zinc-acetate method. volume or, at least, until nitrous fumes are no longer given off. Too great concentrations should be avoided as it of ten results in the precipitation of a very insoluble form of copper and the loss of the determination. Twenty cc. of a saturated solution of zinc acetate, $20 \mathrm{cc}$. of potassium iodide ( $165 \mathrm{~g}$. to $1000 \mathrm{cc}$.) and $60 \mathrm{cc}$. of water are added and the free iodine titrated with $\mathrm{N} /$ Io sodium thiosulfate solution $(24.83 \mathrm{~g}$. per liter). The thiosulfate is run in gradually, with constant shaking, until the brownish yellow color (iodine) has been largely destroyed, then $2 \mathrm{cc}$. of starch paste ( $\mathrm{I} \mathrm{g}$. to $200 \mathrm{cc}$.) are added and the titration continued until the blue particles have entirely disappeared. Towards the end of the reaction the flask should be stoppered and shaken thoroughly.

The copper equivalent of the thiosulfate is determined by diluting $25 \mathrm{cc}$. of a standard copper solution with water, evaporating and titrating exactly as in the test. The standard solution is prepared by dissolving ro grams of pure dry metallic copper in $200 \mathrm{cc}$. of concentrated nitric acid and making up to a liter with water at $20^{\circ} \mathrm{C}$. The solution should be analyzed gravimetrically and will keep almost indefinitely. From this data the reducing action of the sugar solution can be readily calculated in terms of copper and by conversion tables the corresponding amount of sugar. While the first reading of this method might give the impression that it was rather difficult, in reality after a few trials it is extremely simple, can be carried out rapidly and the titration is very sensitive.

1 Eimer and Amend, No. 3263.
E. B. Holland. 